\title{
HETEROGENEITY CORRECTION IN THE CONSTRUCTION OF OPTIMIZED PLANNING IN RADIOTHERAPY USING LINEAR PROGRAMMING
}

\author{
Rodrigo Sartorelo Salemi Viana ${ }^{1 *}$, Helenice de Oliveira Florentino ${ }^{2}$, \\ Ernesto Augusto Bueno da Fonseca Lima ${ }^{3}$, Paulo Roberto da Fonseca ${ }^{4}$ \\ and Thiago Pedro Donadon Homem ${ }^{5}$
}

Received March 19, 2010 / Accepted April 15, 2011

\begin{abstract}
A radiotherapy planning is considered optimal when all the parameters involved, physical or biological, have been investigated and appropriate for each patient. In this type of planning, the major concern is with the tumor irradiation with the minimum possible damage to healthy tissues of the irradiated region, especially the organs at risk. The optimal planning for radiotherapy can be aided by Linear Programming and there is a wide literature addressing this subject. However, most published mathematical formulations do not contemplate a scenario in terms of practical applications, because they do not incorporate the heterogeneous composition of the irradiated tissue. This paper presents a methodology for heterogeneity correction in the composition of different types of irradiated tissues based on proportions among their different linear attenuation coefficient.
\end{abstract}

Keywords: linear programming, optimization, radiotherapy.

\section{INTRODUCTION}

The optimizing theory has been being a mathematical tool of great applicability in the construction of radiotherapy planning, allowing the attainment of results that would for several times

\footnotetext{
*Corresponding author

${ }^{1}$ Instituto de Pesquisas Energéticas e Nucleares, IPEN/CNEN-SP, Av. Lineu Prestes, 2242, Cidade Universitária, São Paulo, SP, Brazil. E-mail: rodrigossviana@gmail.com

${ }^{2}$ Instituto de Biociências de Botucatu, UNESP - Universidade Estadual Paulista, Departamento de Bioestatística, Distrito de Rubião Junior, s/n, Botucatu, SP, Brazil. E-mail: helenice@ibb.unesp.br

${ }^{3}$ Laboratório Nacional de Computação Científica - LNCC, Av. Getúlio Vargas, 333, Quitandinha, Petrópolis, RJ, Brazil. E-mail: eabfl@msn.com

${ }^{4}$ UNIFEB - Centro Universitário da Fundação Educacional de Barretos, Av. Prof. Roberto Frade Monte, 389, Aeroporto, Barretos, SP, Brazil. / Instituto de Biociências de Botucatu, UNESP - Universidade Estadual Paulista, Departamento de Física e Biofísica, Distrito de Rubião Junior SN, Botucatu, SP, Brazil. E-mail: prfonseca@ibb.unesp.br

${ }^{5}$ Serviço Nacional de Aprendizagem Industrial - SENAI, R. Hugo Negrini, 60, Quitandinha, Araraquara, SP, Brazil. E-mail: thiagohomem@yahoo.com.br
} 
be impossible or very hard to be obtained by the use of empiric methods. Among the several parameters involved in the construction of optimized planning, the most commonly explored are related to the positions and relative weightings of the radiation beams used. Several approaches involving different optimization techniques have been applied to radiotherapy planning, among which the integer linear programming, mixed integer and multiobjective (Lee et al., 1999, 2003, 2006; D’Souza et al., 2004; Romeijn, 2003, 2004; Meyer et al., 2003; Yang et al., 2006; Bednarz et al., 2004; Halabi et al., 2006; Schlaefer \& Schweikard, 2008). With the obtained results, the improvement in treatment plans using mathematical techniques stand out, which enabled the development of several technologies and collaborated to the emerging of new equipments for different types of radiotherapy, such as the tomotherapy (Beavis, 2004; Shepard et al., 1999, 2000; Chaudhari et al., 2009; Zhao et al., 2008; Fenwick et al., 2005; Sterpin et al., 2008).

A great concern in the construction process of the treatment plan is the planning simulation, which is not an easy task, for innumerable physical events related to the process of the radiation interaction with matter are, for many times, not considered or are simply not contemplated by the adopted mathematical methodology. The scattered radiation and heterogeneity effects of the irradiated tissues composition (bone, skeletal muscle, and fat tissue, for example) in the calculation of the absorbed dose exemplify these situations. The scattered radiation effect can be studied using non-linear optimization models and the results are coherent with the theory and analytical data reported in the literature (Attix, 1986; Deasy, 1997; Ferris et al., 2003; Wu \& Mohan, (2000); Xing et al., 1998; Spirou \& Chui, 1998), and the heterogeneity effect in the irradiated tissues composition is widely approached in studies using the Monte Carlo method in several cases (Kondratjeva et al., 2006; Tyagi et al. 2008; García et al. 2007; Chetty et al. 2002). However, it is a fact that when considering real problems, the mathematical models to aid the planning are large dimension problems and the non-linear methods for large and medium-scale problems are much more complex than linear programming methods, given the implementation difficulties and computational costs. As to considering the heterogeneity correction by using optimization models, this is still not easily found in the literature. Thus, this work proposes an approach to the heterogeneity correction using penalty factors related to the attenuation for different irradiated media. Aiming to include the physical characteristics in the proposed methodology, a linear programming model formulation was modified.

\section{METHODOLOGY}

\subsection{Optimized treatment plan - a mathematical approach through linear programming}

The first step in developing an optimized treatment plan for radiotherapy consists in defining the location of the tumor, risk organs (critical tissue) and healthy tissues. This procedure is performed with the help of computed tomography images. In this work we used images of real patients obtained with approval from the Research Ethics Committee of the Botucatu Medicine School (process number 3006/2008, approved in 03/11/2008). Through these images, the anatomic regions of interest are selected. 
After the selection of the regions of interest, the computed tomography image is mapped and divided in $m$ pixels. Next, based on mathematical and computational tools like optimization techniques, the deposited dose by each beam is individually calculated considering that each beam is discretized into several beamlets. The modified mathematical model presented in this work was proposed by Holder (2003), who formulated a linear programming model aiming to examine the dosage emitted by beamlets in order to minimize the tissue absorbed dose, restricting the maximum dose to the target tissue and its surrounding regions. The presented methodology proposes the use of this model taking into account the individual dose contribution for different types of tissues, incorporating the heterogeneity effect in the tissue composition.

A realistic mathematical model must consider the radiation beam attenuation, once the deposited dose percentage decreases with the increase in the depth or thickness of irradiated tissue due to interaction mechanisms of the radiation with matter such as absorption, pair production, Compton and Photoelectric scattering. The linear attenuation coefficient $\mu$ quantifies the radiation beam fraction that is absorbed or scattered by thickness unit of the absorbing medium, considering that the different interactions types already mentioned may occur. The sum of the contributions of each process for a given absorbing medium and for a given energy results in the value of $\mu$ (Johns \& Cunningham, 1983).

All the tissues that constitute the human body contribute with some fraction to the attenuation process of the radiation beam. Through the linear attenuation coefficient, the potential dose deposition for these tissues can be explored. Figure 1 shows some values of linear attenuation coefficient for different absorbing mediums (human tissues and air) and energies (Goldstone, 1989).

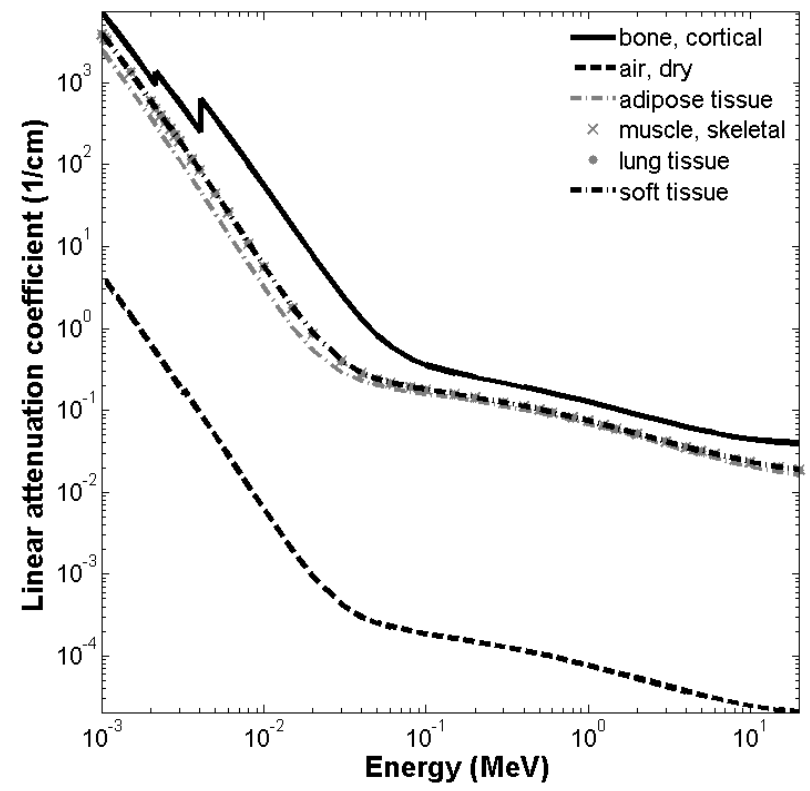

Figure 1 - Linear attenuation coefficients for different absorbing mediums. 
According to the Figure 1, the proximity between the linear attenuation coefficients referring to tissues such as lung, skeletal muscle and adipose tissue can be observed, especially with the energy increase. Conventionally, blood, arteries, veins, lungs, adipose tissue, skeletal muscle and others are recognized as soft tissue (Goldstone, 1989). Under this approach, three main structures are considered: soft tissue, cortical bone and air cavities, which present distinct values of linear attenuation coefficients. This differentiation is fundamental to the study of the dose deposition on the irradiated tissue that underlies the mathematical model (Attix, 1986). Considering a $10 \mathrm{MV}$ photons beam, the ratio between the linear attenuation coefficients to air, soft tissue and cortical bone, respectively, is $1.06 \mathrm{E}-3: 1: 1.91$. It is proposed to use this ratio as additional penalty factor in constraints of the mathematical model to be discussed next.

The model proposed by Holder (2003) uses $k$ angular positions for beams emission: $\theta_{1}, \theta_{2}$, $\theta_{3}, \ldots, \theta_{k}$ where each angle is comprised of $\eta$ beamlets. Modern systems of treatment are capable of performing complex combinations among these beamlets and use them along all the angular variation, making the planning use $k \cdot \eta$ beamlets at the most.

We set $x_{(a, i)}$ to be the dose along the $i^{\text {th }}$ beamlet $(i=1,2, \ldots, \eta)$ of the $a^{\text {th }}$ angle $(a=$ $1,2, \ldots, k)$ and $d_{(p, a, i)}$ to be the distance from the positioned source on the angle emitting the beamlet $i$ (of dose $\left.x_{(a, i)}\right)$ and pixel $p(p=1,2, \ldots, m)$. Dose deposition for pixel $p$ due to the beamlet $i$ of the angle $a, A_{(p, a, i)}$, is defined:

$$
A_{(p, a, i)}=e^{-\mu \cdot d_{(p, a, i)}} A_{x p} .
$$

The terms $A_{x p}$ and $e^{-\mu \cdot d_{(p, a, i)}}$ represent the geometric area of the pixel $p$ that receives the dose $x_{(a, i)}$ and the attenuation factor, respectively. As the used images are related to real cases of planning, to make more realistic simulations, the attenuation factor was replaced by values obtained in calibration of a $10 \mathrm{MV}$ photons beam on water, once this is the main constituent of the human body and a standard dosimetric procedure. For the proposed approach, equation 1 was modified by the inclusion of the parameter $f(p)$ that relates the location of the pixel $p$ with the composition of the irradiated tissue. Therefore, equation 1 becomes:

$$
A_{(p, a, i)}=f(p) e^{-\mu \cdot d_{(p, a, i)}} A_{x p}
$$

The parameter $f(p)$ takes a value in the ratio $1.06 \mathrm{E}-3: 1: 1.91$ for air, soft tissue and cortical bone, respectively, according to the localization of pixel $p$, and this way, the equation 2 linearly considers the radiation beam attenuation profile as well as the heterogeneous composition of the irradiated medium. The tissues classification may be implemented using statistical methods of images segmentation such as the Gaussian mixture method, used in this work, in which the Gaussian parameters are estimated through the EM algorithm (Expectation Maximization) (Dempster et al., 1977; Yamazaki \& Yamazaki, 1998). With the components of $A_{(p, a, i)}$ it is possible to construct the dose deposition matrix $\mathbf{A}$, where the rows of $\mathbf{A}$ are indexed by $p$ and the columns are indexed by $(a, i)$. 
Let $m_{T}$ be the number of target pixels, $m_{C}$ be the number of critical structure pixels, and $m_{G}$ be the number of healthy tissues pixels, so the total number of pixels is $m=m G+m T+m C$. Then, we can assume dose deposition matrix $\mathbf{A}$ as indicated below:

$$
A=\left[\begin{array}{l}
A_{T} \\
A_{C} \\
A_{G}
\end{array}\right],
$$

where the rows of the dose deposition matrix are reordered so that $T$ corresponds to the set of pixels encompassing tumors, $C$ to the set of pixels encompassing critical tissues or risk organs and $G$ to the set of pixels encompassing healthy tissues.

Let $\mathbf{x}$ be the vector of dose, whose components are given by the beamlets $x_{(a, i)}$, the total dose radiation for pixel $p$ is given by the $p^{\text {th }}$ component of the vector resulting from the multiplication Ax. As mentioned, in the treatment using radiotherapy a limiting dose is prescribed for each kind of tissue (tumor, critical and healthy). Due to the pixel division, in the model that will be presented, dose prescription will be given through vectors, as following:

ULT: represents the vector of upper bounds for tumor radiation dose (ULT $\in \Re^{m_{T}}$ ),

LLT: represents the vector of lower bounds for tumor radiation dose $\left(\mathbf{L L T} \in \mathfrak{R}^{m_{T}}\right)$,

ULC: represents the vector of upper bounds for critical tissue radiation dose (ULC $\in \Re^{m_{C}}$ ),

ULG: represents the vector of upper bounds for healthy tissue radiation dose (ULG $\in \mathfrak{R}^{m}{ }_{G}$ ).

Considering that $0 \leq L L T \leq U L T, U L C \geq 0$ and $U L G \geq 0$. Assuming that a $T_{P}$ dose was established for cancer cells, the values of ULT and LLT are usually $(1+\varepsilon) T_{P}$ and $(1-\varepsilon) T_{P}$, respectively, where $\varepsilon$ is the variation percentage for tumor dosage and is called tumor uniformity level. Typical values of $\varepsilon$ found in literature range from $2 \%$ to $15 \%$. In the model studied, dose prescription is peculiar to each kind of treatment, taking into consideration the development level of the disease, the tumor volume and mainly the anatomy of the irradiated region.

Defined these parameters, the formulation of the linear programming problem proposed in Holder (2003) is of the form:

$$
\begin{array}{cl}
\text { minimize } & l^{T} t+u_{c}^{T} c+u_{g}^{T} g \\
\text { subject to: } & \\
& L L T-I_{L} t \leq A_{T} x \leq U L T \\
& A_{C} x \leq U L C+I_{C} c \\
& A_{G} x \leq U L G+I_{G} g \\
& 0 \leq I_{L} t \leq L L T \\
& -U L C \leq I_{C} c \\
& I_{G} g \geq 0 \\
& x \geq 0 .
\end{array}
$$


The model (4-11) consists of determining the vector $\mathbf{x}$ corresponding to the relative weights (or modulated doses) of beamlets employed in the planning, as well as the vectors $\mathbf{t}, \mathbf{c}$ and $\mathbf{g}$ that satisfy the dose limitation constraints by the type of irradiated structure and that optimize an objective function, $\mathbf{x} \in \Re^{k \cdot \eta}, \mathbf{t} \in \Re^{m_{T}}, \mathbf{c} \in \Re^{m_{C}}$ e $\mathbf{g} \in \Re^{m_{G}}$. The vectors $\mathbf{l}, \mathbf{u}_{\mathbf{c}}$ and $\mathbf{u}_{\mathbf{g}}$ are respectively defined as $\left(1 / \mathrm{m}_{\mathrm{T}}\right) e,\left(1 / \mathrm{m}_{\mathrm{C}}\right) e$ e $\left(1 / \mathrm{m}_{\mathrm{G}}\right) e$, where e denotes a vector of ones and possesses appropriate dimensions. The matrices $\mathbf{I}_{\mathbf{L}}, \mathbf{I}_{\mathbf{C}}$ and $\mathbf{I}_{\mathbf{G}}$ are defined as identity, in which $\mathbf{I}_{\mathbf{L}} \in \Re^{m_{T} m_{T}}, \mathbf{I}_{\mathbf{C}} \in \Re^{m_{C} m_{C}}$ and $\mathbf{I}_{\mathbf{G}} \in \Re^{m_{G} x m_{G}}$. The objective is given by adding three goals for the treatment: $l^{T} t+u_{c}^{T} c+u_{g}^{T} g$, where:

$l^{T} t$ : deficit of dose applied to the tumor with respect to the prescribed dose, $u_{c}^{T} c$ : excess of dose applied to the critical tissue with respect to the prescribed dose, $u_{g}^{T} g$ : excess of dose applied to the healthy tissue with respect to the prescribed dose.

The constraints (5), (6) and (7) are called elastic because the bounds are allowed to vary with vectors $\mathbf{t}, \mathbf{c}, \mathbf{g}$ and $\mathbf{x}$, which correspond to the variables of the problem. The matrices $\mathbf{I}_{\mathbf{L}}, \mathbf{I}_{\mathbf{C}}$ and $\mathbf{I}_{\mathbf{G}}$ define how one measures the amount of elasticity, and $\mathbf{l}, \mathbf{u}_{\mathbf{c}}$ and $\mathbf{u}_{\mathbf{g}}$ show how one either penalizes or rewards the amount of elasticity. These elastic functions are incorporated into the problem to ensure that the set of constraints is always strictly feasible (Berman \& Plemmons 1987).

The simulations were performed on a computer with $2.4 \mathrm{GHz}$ Intel ${ }^{\circledR}$ QuadCore $^{\mathrm{TM}}$ and $4 \mathrm{~GB}$ RAM desktop in the MACBAM Laboratory of the Biostatistics Department of the Biosciences Institute - UNESP. The linear programming problem was solved using the GLPK software (GNU Linear Programming Kit, source code available free at http://www.gnu.org/software/glpk, Linux operating system) and the selection of regions of interest, processing and visualization of the optimized level curves were performed using the MATLAB software (2007a, The MathWorks Inc., Natick, MA).

Considering the approach proposed in this paper, a brief resume of the procedure for the creation of optimized planning is showed in Figure 2.

\section{RESULTS AND DISCUSSION}

For the performed simulations three computed tomography images of real patients were used, in which well-defined tumor masses were diagnosed. Figure 3 shows the images used in the simulations.

In the 3-A image multiple tumor masses were diagnosed in the liver and were considered as critical tissues the kidneys and the bone marrow. In the 3-B image two tumor masses were observed in the lung, being the bone marrow considered as critical tissue. In the image 3-C the tumor mass is located in the right kidney and the bone marrow, left kidney and liver were considered critical tissues.

For the simulations, 8 angular positions were used $\left(k=0^{\circ}, 45^{\circ}, 90^{\circ}, 135^{\circ}, 180^{\circ}, 225^{\circ}, 270^{\circ}\right.$, $\left.315^{\circ}\right)$ and 1792 beamlets $(k \cdot \eta=1792)$. Without loss of generality, the dose prescription was assumed as non-dimensional and the values adopted for the vectors elements ULT, LLT, ULC 


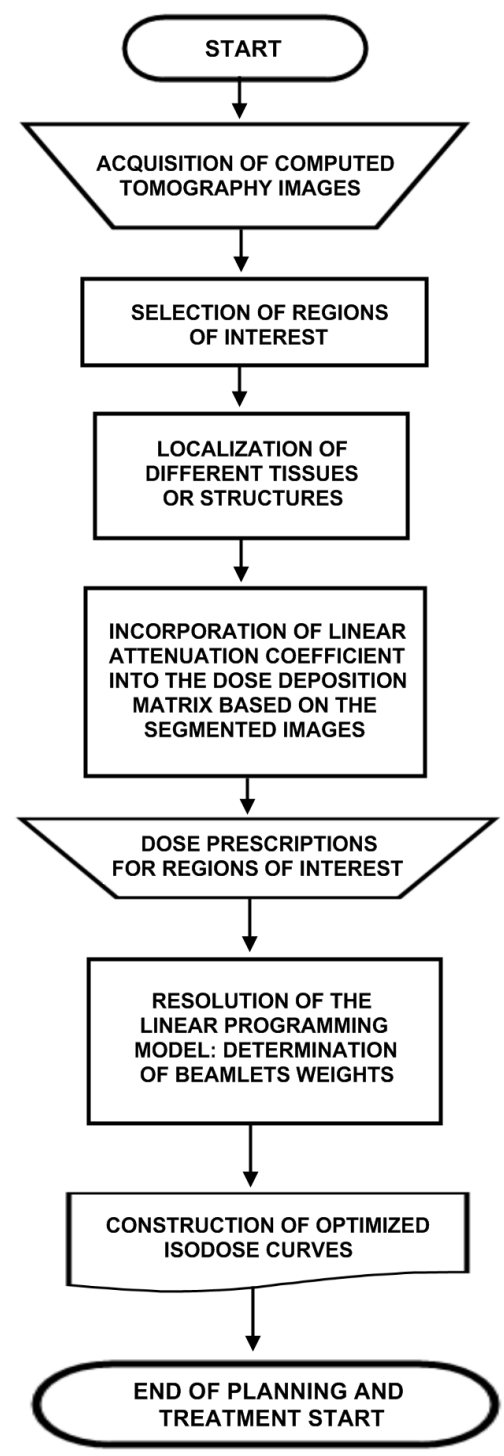

Figure 2 - Flowchart of the optimized planning considering the localization of regions of interest and irradiated tissues composition.

and ULG were respectively $100,90,36$ and 38 . The dose prescriptions proposed in this paper take into consideration a situation where is desired a high dose deposition at the tumor and a low dose deposition at the other tissues.

As described, the modification in the proposed model by Holder (2003) is based on different levels of attenuation of the human body tissues, which can be expressed numerically by the linear attenuation coefficient. Regardless of any technique for heterogeneities correction, the attenuation coefficient is a parameter in common and physically expresses the capacity of irradiated 


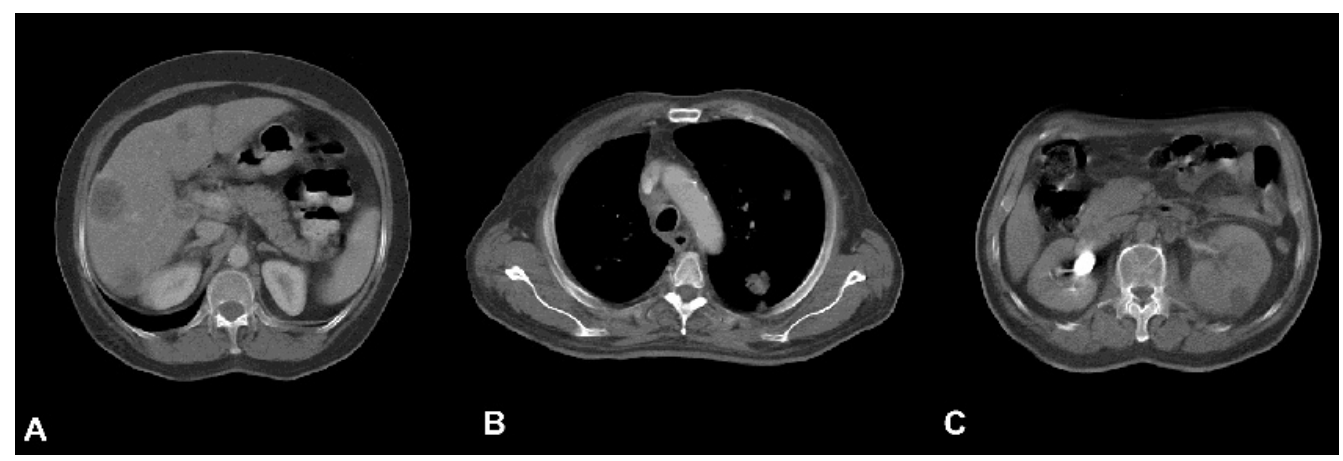

Figure 3 - Computed tomography images used in the simulations.

tissues in absorbing radiation (Attix, 1986). Another important fact about the used methodology is the proper classification of different types of tissues or structures.

The grayscale images produced by computed tomography images reconstruction algorithms are normalized with the linear attenuation coefficient of water. Thus, regions well defined by gray shades are reliably associated with structures, biological or not, of different radiopaque properties. Therefore, bone tissue and air cavities are always assigned with gray shades at the extremes of the color scale image. In this way, the tissues selection as well as its heterogeneous composition relate with the linear attenuation coefficient in the proposed methodology.

Two types of planning were carried out: the first planning was made using the methodology proposed in this work considering the heterogeneity correction, Figure 4, and for comparison, the second planning was made based exclusively upon the methodology proposed in Holder (2003) which does not include such correction, Figure 5.

Figure 4 shows on the left side the tomography images $\mathrm{A}, \mathrm{B}$ and $\mathrm{C}$ with demarcation of tumor regions (full lines) and critical tissues (dashed lines), and the optimized plannings obtained using the methodology discussed in this work by means of isodose curves (contour lines with respect to the optimized dose) for each case. The optimized plans were done considering a $100 \times 100$ image grid. The computing performance and the terms of the objective function (equation 4) of the model for both types of planning are shown in Table 1 .

Table 1 - Minimized values for the three goals that compose the objective function considering the two approaches.

\begin{tabular}{|c|c|c|c|c|c|}
\hline Approach & Image & $l^{T} t$ & $u_{c}^{T} c$ & $u_{g}^{T} g$ & CPU time (s) \\
\hline \multirow{4}{*}{ With heterogeneity correction } & $\mathrm{A}$ & $1.54 \mathrm{E}-2$ & 0 & 0 & 46.40 \\
& $\mathrm{~B}$ & $1.40 \mathrm{E}-2$ & 0 & 0 & 47.55 \\
& $\mathrm{C}$ & $0.98 \mathrm{E}-2$ & 0 & 0 & 44.17 \\
\hline \multirow{5}{*}{ Without heterogeneity correction } & $\mathrm{A}$ & $2.93 \mathrm{E}-2$ & 0 & 0 & 54.32 \\
& $\mathrm{~B}$ & $2.77 \mathrm{E}-2$ & 0 & 0 & 55.72 \\
& $\mathrm{C}$ & $2.09 \mathrm{E}-2$ & 0 & 0 & 51.25 \\
\hline
\end{tabular}


An apparent characteristic of the results presented in Figure 4 is the conformation of the maximum dose on the tumor region and a low dose deposition on critical and healthy tissues, once the dose limits prescribed for these tissues were respected. Considering the critical tissues, their localization did not compromise the dose conformation in the tumor even when relatively close to the target (Figs. 4-A and 4-C). The same behavior can also be observed in Figure 4-B, where the presence of air cavities (lungs) also did not alter the dose deposition profile.

Using the same simulation parameters, the planning without heterogeneity correction was performed considering only the position of the regions of interest. As expected, the results obtained with respect to the isodose curves and to the values of terms of the objective function of the model (4-11) were different, Table 1.

Figure 5 shows the isodose curves for optimized planning without heterogeneity correction. Aiming to qualitatively compare the methodologies involved in the two approaches, the proportion between isodose regions areas for planning with and without heterogeneities correction was obtained. The optimized planning must ensure that the prescribed dose is delivered to the tumor while the deposited dose in healthy tissues and critical is the lowest possible. Thus, according to the proportion between isodose regions for images $\mathrm{A}, \mathrm{B}$, and $\mathrm{C}$, it is possible to observe that the extension of the isodose curves referring to 30,40 and 50 in planning without heterogeneity correction is slightly higher compared with the isodose curves obtained with the proposed methodology. Furthermore, given the position of the structures of interest and the dose prescription, when the heterogeneity correction is not considered the performed planning was not successful in three cases with respect to dose delivery at the prescribed upper limit to the tumor $(\mathrm{LLT}=100)$.

In both approach types, the planning was successful with respect to the dose delivery within the prescribed dose limits to the tumor. However, when considering the heterogeneity correction, the minimized value of the objective function was lower when compared to the second planning (Table 1). Another fact to be observed is the difference of the cpu time involved in the two approaches.

The proposed methodology implementation resulted in a gain in cpu time about $14 \%$. It must be considered that real planning for radiotherapy have much larger dimensions than the ones adopted in this work. Specifically, the amount of assessed pixels by image, number of angular combinations for the radiation beams emissions and the number of beamlets used in each angular position demand a high level computing performance (Beavis, 2004).

\section{CONCLUSION}

In both planning types, an apparent characteristic in the results is the conformation of the maximum dose only on the target region. In cases of multiple tumor foci or with the presence of air cavities the optimized planning was successful both in the dose delivery within the prescribed dose limits to the tumor, critical, and healthy tissues. However, given the position of the structures of interest and the dose prescription, when the heterogeneity correction is not considered the performed planning was not successful with respect to dose delivery at the prescribed upper 

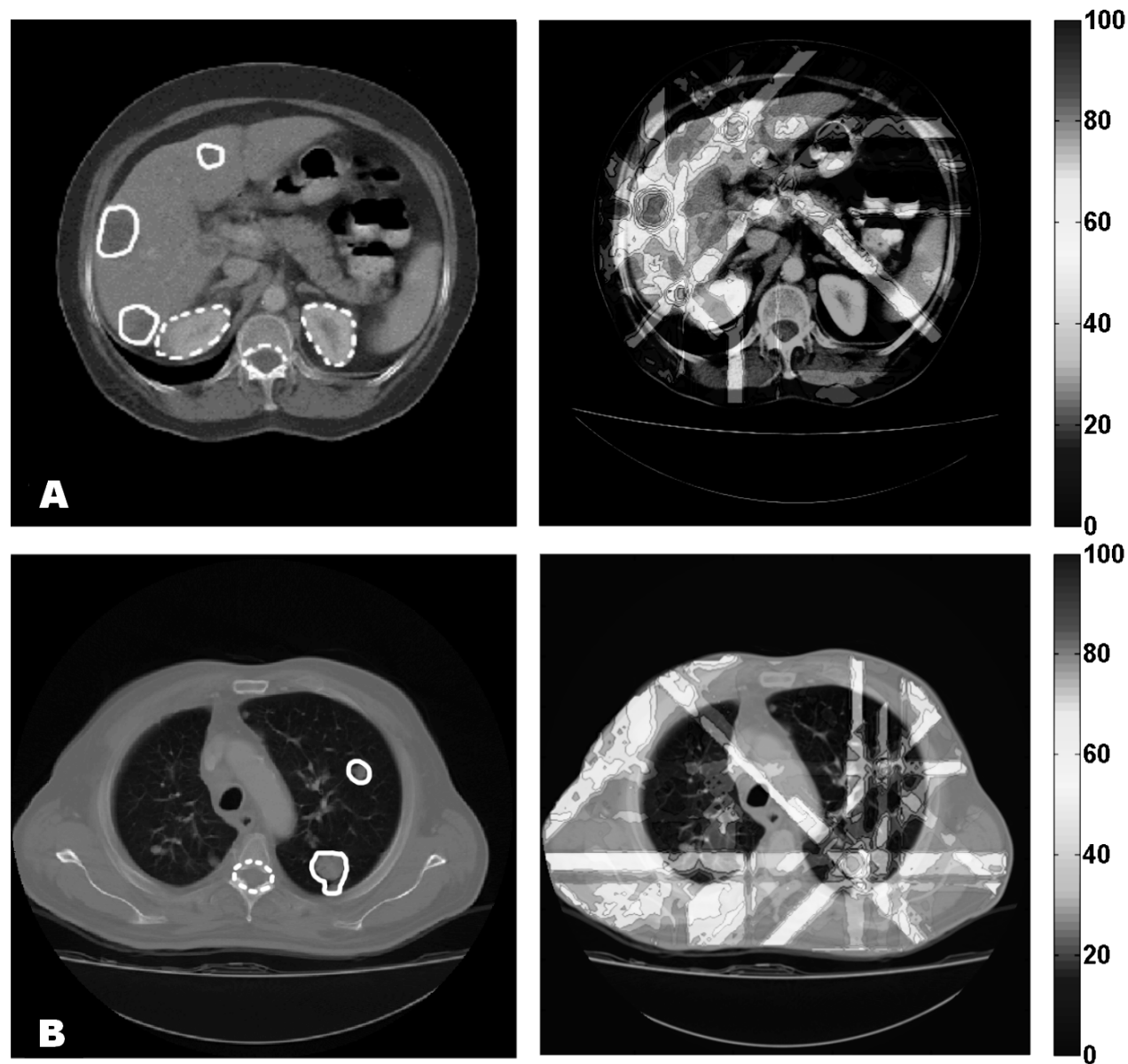

$-80$

$-60$

$-40$

20
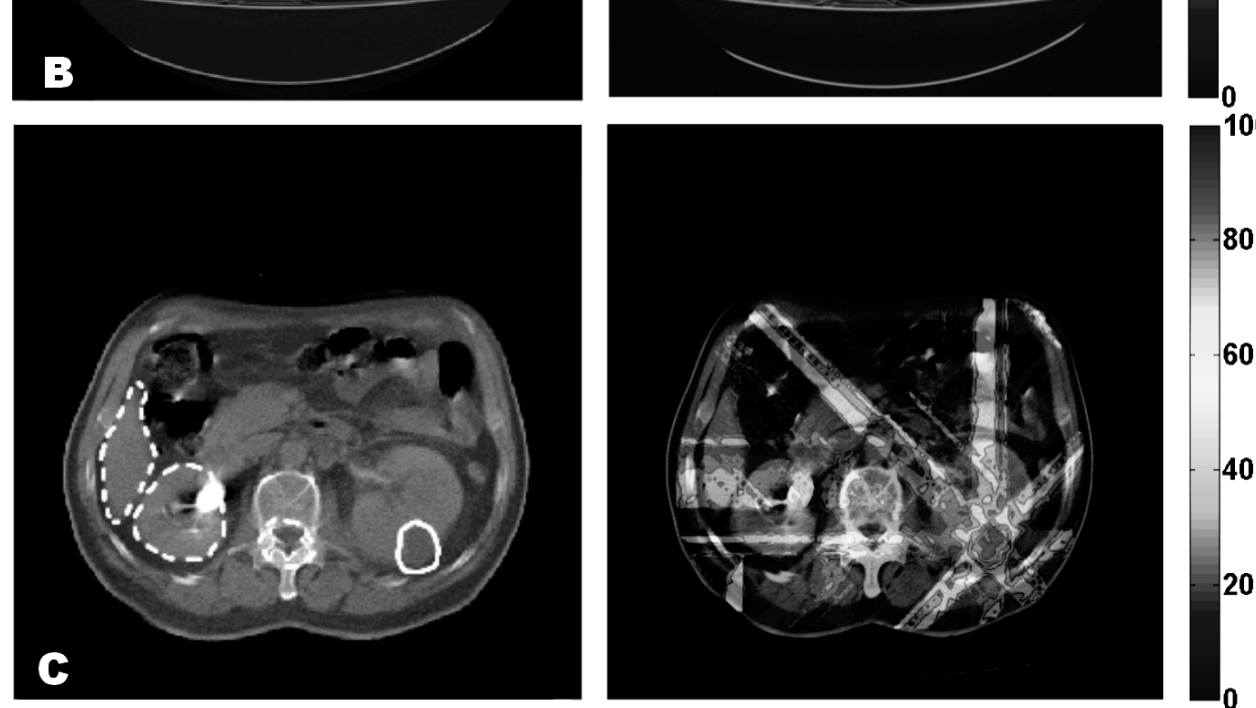

Figure 4 - Computed tomography images used in the simulations. The detached regions with full lines represent the tumor location and the detached regions with dashed lines represent the critical tissues locations. Results obtained with the proposed methodology. 

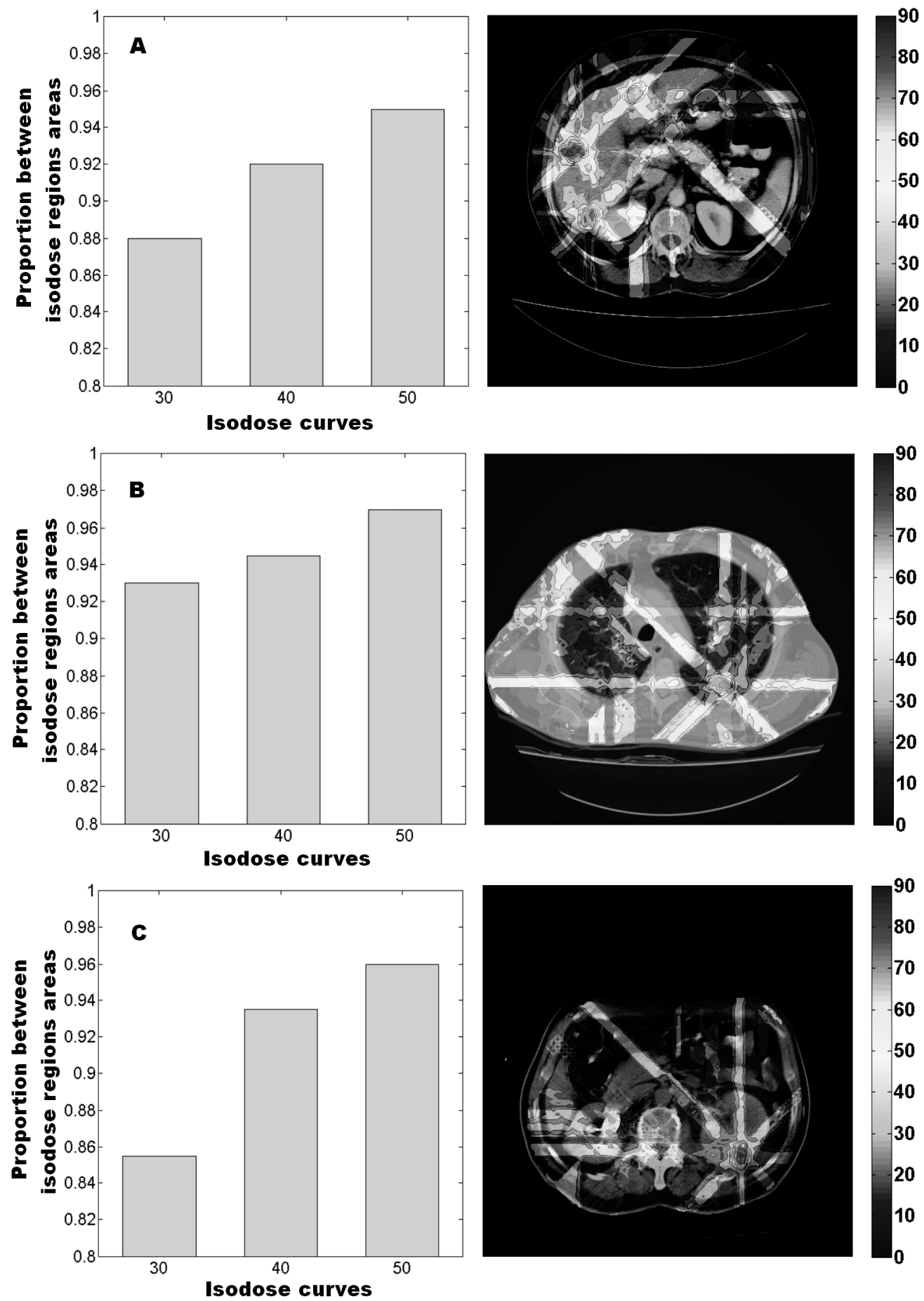

$-70$
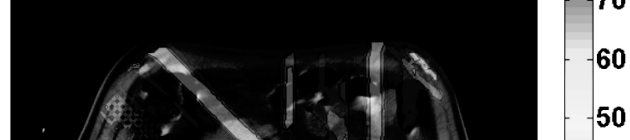

50

$-40$

$-30$

20

10

Figure 5 - Proportion between isodose regions areas, respectively for the images A, B and C. Isodose curves for the optimized planning without considering the heterogeneity correction. 
limit to the tumor and the isodose curves extent are larger compared with the planning performed with the proposed methodology. Assuming that this behavior was seen in low dose (30, 40 and 50 ), even if the difference is small, healthy and critical tissues are getting an undesirable dose delivery, this same logic can be extended to the difference between the minimized values of the objective function.

Considering a linear programming model, the approach used in this paper allowed more coherent interpretation, once that this methodology can be applied to various types of tissues or structures, which would make a more detailed modeling, because the tissues heterogeneous composition is linearly incorporated in the mathematical modeling. The majority of already published works addresses, by different means, the obtaining of the best composition of relative weights for the beamlets considering basically the initial location of the structures of interest and dose prescription. However, the irradiated tissues composition is not incorporated to the mathematical formulations, even being extremely important. Thus, this work may be seen as collaboration to future formulations of linear programming models through a procedure to incorporate different levels of attenuation of the human body tissues.

\section{ACKNOWLEDGEMENTS}

The authors thank the financial support from: FUNDUNESP (Fundação para o Desenvolvimento da UNESP), FAPESP (Fundação de Amparo à Pesquisa do Estado de São Paulo, grant no 07/02313-5, 06/02476-9 and 05/56251-5) and PROPe (Pró-Reitoria de Pesquisa da UNESP).

\section{REFERENCES}

[1] AtтiX F. 1986. Introduction to Radiological Physics and Radiation Dosimetry. Wiley-Interscience, USA.

[2] BeAVIS A. 2004. Is tomotherapy the future of IMRT? Br J Radiol, 77: 285-295.

[3] Bednarz G, Michalski D, Anne P \& Valicenti R. 2004. Inverse treatment planning using volume-based objective functions. Phys Med Biol, 49(12): 2503-2514.

[4] Berman A \& Plemmons R. 1987. Nonnegative matrices in the mathematical sciences. Society for Industrial Mathematics, USA.

[5] Chaudhari S, Pechenaya O, Goddu S, Mutic S, Rangaraj D, Bradley J \& Low D. 2009. The validation of tomotherapy dose calculations in low-density lung media. Phys Med Biol, 54(8): 2315-22.

[6] Chetty I, Moran J, Nurushev T, McShan D, Fraass B, Wilderman S \& Bielajew A. 2002. Experimental validation of the DPM Monte Carlo code using minimally scattered electron beams in heterogeneous media. Phys Med, 47: 1837-1851.

[7] Deasy J. 1997. Multiple local minima in radiotherapy optimization problems with dose-volume constraints. Med Phys, 24(7): 1157-1161.

[8] Dempster A, Laird N \& Rubin D. 1977. Maximum Likelihood from Incomplete Data via the EM Algorithm. J Roy Stat Soc B Met, 39: 1-38. 
[9] D'Souza W, Meyer R \& Shi L. 2004. Selection of beam orientations in intensity-modulated radiation therapy using single-beam indices and integer programming. Phys Med Biol, 49(15): $3465-3481$.

[10] Fenwick J, TOMÉ W, Kissick M \& MACKIE R. 2005. Modelling simple helically delivered dose distributions. Phys Med Biol, 50: 1505-1517.

[11] Ferris M, Lim J \& Shepard D. 2003. Radiosurgery Treatment Planning via Nonlinear Programming. Ann Oper Res, 119: 1-4.

[12] García E, Jiménez J \& Puimedón J. 2007. Dose calculation in patients with PENELOPE/ PENGEOM. J Phys Conf Ser, 74021006 (4pp) doi: 10.1088/1742-

[13] Goldstone K. 1989. Tissue Substitutes in Radiation Dosimetry and Measurement, Report 44 of the International Commission on Radiation Units and Measurements (Bethesda, MD).

[14] Halabi T, Craft D \& Bortfeld T. 2006. Dose-volume objectives in multi-criteria optimization. Phys Med Biol, 51(15): 3809-3818.

[15] Holder A. 2003. Designing radiotherapy plans with elastic constraints and interior point methods. Health Care Management Science, 6: 5-16.

[16] Johns H \& Cunningham J. 1983. The Physics of Radiology. Charles C. Thomas Publisher, USA.

[17] Kondratjeva A, Kolchuzhin A, Lisin V \& Tropin I. 2006. Properties of absorbed dose distribution in heterogeneous media. J Phys Conf Ser, 41: 527-530.

[18] Lee E, Fox T \& Crocker I. 2006. Simultaneous beam geometry and intensity map optimization in intensity-modulated radiation therapy. Int J Radiat Oncol Biol Phys, 64(1): 301-320.

[19] Lee E, Fox T, Crocker I \& Burjony M. 2003. Integer programming applied to intensitymodulated radiation therapy treatment planning. Ann Oper Res, 119: 165-181.

[20] Lee E, Gallagher R, Silvern D, Wuu C \& Zaider M. 1999. Treatment planning for brachytherapy: an integer programming model, two computational approaches and experiments with permanent prostate implant planning. Phys Med Biol, 44(1): 145-165.

[21] Meyer R, D’Souza W, Ferris M \& Thomadsen B. 2003. MiP models and BB strategies in brachytherapy treatment optimization. J Glob Optim, 25: 23-42.

[22] Romeijn H, Ahuja R, Dempsey J, Kumar A \& Li J. 2003. A novel linear programming approach to fluence map optimization for intensity modulated radiation therapy treatment planning. Phys Med Biol, 48: 3521-3542.

[23] Romeijn H, Dempsey J \& Li J. 2004. A unifying framework for multi-criteria fluence map optimization models. Phys Med Biol, 49(10): 1991-2013.

[24] Schlaefer A \& SchweiKard A. 2008. Convex reformulation of biologically-based multi-criteria intensity-modulated radiation therapy optimization including fractionation effects. Phys Med Biol, 53(22): 6345-6362.

[25] Shepard D, Ferris M, Olivera G \& Mackie R. 1999. Optimizing the delivery of radiation therapy to cancer patients. SIAM Review, 41: 721-744.

[26] Shepard D, Olivera G, Reckwerdt P \& Mackie R. 2000. Iterative approaches to dose optimization in tomotherapy. Phys Med Biol, 45: 69-90. 
[27] SPIROU S \& CHUi C. 1998. A gradient inverse planning algorithm with dose-volume constraints. Med Phys, 25(3): 321-333.

[28] Sterpin E, Salvat F, Cravens R, Ruchala K, Olivera G \& Vynckier S. 2008. Monte Carlo simulation of helical tomotherapy with PENELOPE. Phys Med Biol, 53(8): 2161-2180.

[29] Tyagi N, Curran B, Roberson P, Moran J, Acosta E \& Fraass A. 2008. Experimental verification of a Monte Carlo-based MLC simulation model for IMRT dose calculations in heterogeneous media. J Phys Conf Ser, 102: 1-8.

[30] Wu Q \& Mohan R. 2000. Algorithms and functionality of an intensity modulated radiotherapy optimization system. Med Phys, 27(4): 701-711.

[31] Xing L, Hamilton R, Spelbring D, Pelizzari C, Chen G \& Boyer A. 1998. Fast iterative algorithms for three-dimensional inverse treatment planning. Med Phys, 25: 1845-1849.

[30] YAMAZAKI T \& YAMAZAKI T. 1998. Introduction of EM Algorithm into Color Image Segmentation. Proc. ICIPS'98, (Institute of Electrical and Electronics Engineers: International Conference on Intelligent Processing Systems Proceedings), 368-371.

[33] YANG R, DAi J, YANG Y \& HU Y. 2006. Beam orientation optimization for intensity-modulated radiation therapy using mixed integer programming. Phys Med Biol, 51: 3653-3666.

[34] Zhao Y, Mackenzie M, Kirkby C \& Fallone B. 2008. Monte Carlo calculation of helical tomotherapy dose delivery. Med Phys, 35(8): 3491-3500. 